\title{
Degradación de T-patterns en estudios observacionales: Un estudio sobre la eficacia en el ataque de fútbol sala
}

\author{
Degradation of T-patterns in observational studies: \\ A study on the effectiveness in futsal
}

\section{Degradação de t-patterns em estudos observacionais: Um estudo sobre a eficácia no futsal}

\author{
Daniel Lapresa ${ }^{1}$, Oleguer Camerino ${ }^{2}$, Josep Cabedo ${ }^{3}$, M. Teresa Anguera ${ }^{4}$, Gudberg K. Jonsson y Javier Arana ${ }^{6}$ \\ ${ }^{1}$ Universidad de La Rioja (España), ${ }^{2}$ INEFC-Universidad de Lleida (España), ${ }^{3}$ Universidad Ramon Llull (España), ${ }^{4}$ Universidad de Barcelona (España), \\ ${ }^{5}$ University of Iceland (Iceland), ${ }^{6}$ Universidad Internacional de La Rioja (España)
}

\begin{abstract}
Resumen: Una de las técnicas analíticas más novedosas en el ámbito de la metodología observacional es la que permite la detección de T-patterns (estructuras regulares de conducta) mediante el software THEME, que analiza datos observacionales en búsqueda de relaciones de intervalo crítico en sentido ascendente (desde T-pattern simples, dos eventos que se relacionan en un primer nivel, a T-patterns complejos constituidos por diferentes eventos relacionados en distintos niveles). En el presente artículo se analiza esta cuestión, la degradación de los T-patterns, que hasta el momento ha sido puntualmente estudiada. Para ello, se utiliza el muestreo observacional constituido por el conjunto de las secuencias que acaban en gol $(n=23)$ protagonizadas por el F.C. Barcelona durante el Play Off final de la liga española 2012-13 de fútbol sala. De esta forma este trabajo satisface un segundo objetivo, en este caso disciplinar, de analizar la eficacia en la construcción de la fase ofensiva en el fútbol sala; habiéndose tipificado que las secuencias que finalizan en gol se caracterizan por conseguir, mediante juego combinativo, un lanzamiento desde sector definición-corredor central.

Palabras claves: Metodología observacional, fútbol sala, T-patterns, THEME, degradación.

Abstract: One of the most recent analytical techniques in observational methodology is T-pattern detection and analysis (regular structures of behaviour) with the THEME software. THEME analyses observational data, searching for critical interval relationship between single event types to more complex T-pattern structures. The current study analyses the degradation of T-patterns that have only been studied punctually. With this objective we analyse a sample of sequences from Barcelona FC, from the final
\end{abstract}

playoffs in the Spanish Futsal League, that end in a goal $(n=23)$. A second objective of this study is to analyse the efficacy of offensive phase in futsal, it having been typified that the sequences that they finish in goal characterize for central definition - broker obtains, by means of game combinativo, a throwing from sector.

Keywords: Observational methodology, futsal, T-patterns, THEME, degradation.

Resumo: Uma das técnicas analíticas mais recentes no âmbito da metodología observacional é a que permite a deteção de T-patterns (estruturas regulares de conduta) através do software THEME. THEME analisa dados observacionais em busca de relaçóes de intervalo crítico no sentido ascendente (a partir dos T-pattern simples, dois eventos que se relacionan num primeiro nivel, até T-patterns complexos constituidos por diferentes eventos que se relacionams em diferentes níveis). No presente artigo analisa-se esta questấo -a degradação dos T-patterns- que até ao momento foi estudada de forma pontual. Para isso, utiliza-se a amostragem observacional constituída pelo conjunto de sequências que terminam em golo $(n=23)$ protagonizadas pelo F.C. Barcelona durante o Play Off final da liga espanhola correspondente à temporada 2012-13. Desta forma este trabalho satisfaz um segundo objetivo, neste caso disciplinar, de analisar a eficácia na construção da fase ofensiva no futsal, tendo estabelecido que as sequências que terminam em gol caracterizam-se por alcançar, usando o jogo combinatório, um lançamento do sector definicion-corredor central.

Palavras chave: metodologia observacional, futsal, T-patterns, THEME, degradação.

\section{Introducción}

La metodología observacional, que se desarrolla en contextos naturales o habituales, consiste en un procedimiento científico que pone de manifiesto la ocurrencia de conductas perceptibles, para proceder a su registro organizado y su análisis -tanto cualitativo como cuantitativo- mediante un instru-

Dirección para correspondencia [Correspondence address]: Daniel Lapresa Ajamil. Universidad de La Rioja. Departamento de Ciencias de la Educación. Edificio Vives, C/ Luis de Ulloa s/n. 26004. Logrońo, La Rioja (España). E-mail: daniel.lapresa@unirioja.es mento adecuado y parámetros convenientes, posibilitando la detección de las relaciones de diverso orden existentes entre ellas y evaluándolas. Estas conductas, por la espontaneidad o habitualidad con que ocurren, pondrán de manifiesto todos aquellos elementos que se requiere destacar para alcanzar su objetivación adecuada. La incidencia de la metodología observacional en la investigación sobre la psicología del deporte ha sido considerable en la última década, su utilización como método de análisis y los avances tecnológicos y estadísticos 
que ha provocado marcan un desarrollo emergente de la utilización de la metodología observacional en la psicología del deporte (Anguera y Hernández-Mendo, 2014).

Una de las técnicas de visibilización de estructuras regulares de conducta de mayor repercusión (Camerino, Chaverri, Anguera y Jonsson, 2012; Camerino, Prieto, Lapresa, GutiérrezSantiago e Hileno, 2014; Lapresa, Arana, Anguera y Garzón, 2013; Zurloni, Cavalera, Diana, Elia y Jonsson, 2014), se lleva a cabo mediante el software THEME (Magnusson, 1996, 2000, 2005, 2006), que permite la detección de patrones temporales o T-patterns. Un T-pattern es una "construcción esta- dística” que se manifiesta a partir de una combinación de eventos, que ocurren en el mismo orden con distancias temporales entre sí (intervalo crítico) que se mantienen relativamente invariantes en relación a la hipótesis nula de que cada código de conducta registrado es independiente y está distribuido aleatoriamente en el tiempo. De acuerdo con Magnusson (2000, p. 94-95), "si A es el componente anterior y B el posterior de un mismo patrón temporal que se repite, entonces después de una ocurrencia de $A$ en el tiempo $t$, hay un intervalo $[t+d 1, t+d 2]$ $(\mathrm{d} 2 \geq \mathrm{d} 1 \geq \mathrm{d} 0)$ que tiende a contener al menos una ocurrencia de B más a menudo de lo que se esperaría por azar".

Figura 1. Representación gráfica del proceso de detección de T-patterns (Magnusson, 2000, p. 94-95).

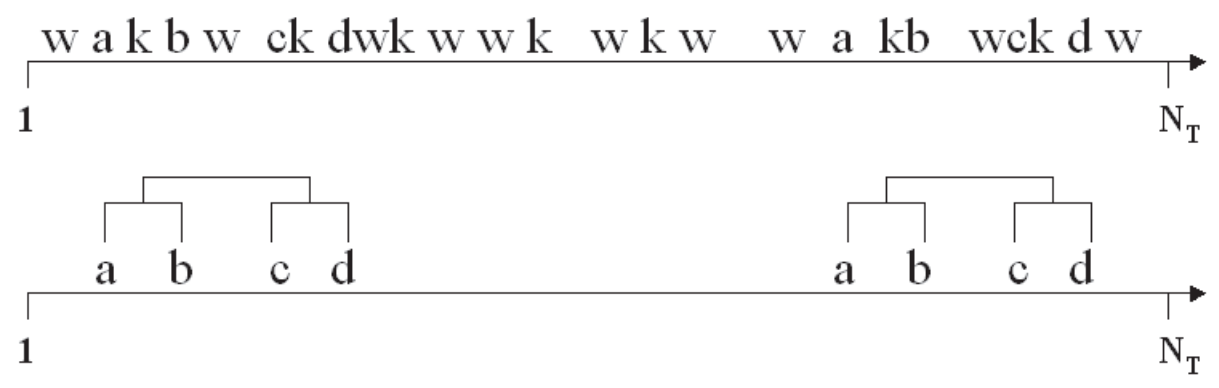

De esta forma, cuando THEME halla que una ocurrencia de A es seguida por una de $\mathrm{B}$, dentro de un intervalo crítico, se genera un T-pattern simple (AB). En este caso (véase figura 1), nos encontramos con un T-pattern de primer nivel de relación y de 2 clusters $^{1}$. Otros T-patterns pueden encontrarse relacionados con el patrón simple de partida (AB), ya que THEME repite el procedimiento de detección nivel a nivel (de 1 hasta $n$ ), buscando relaciones de intervalo crítico que, en su seno, incorporen a los patrones temporales ya detectados. De esta forma, se pueden encontrar relaciones de intervalo crítico entre T-patterns simples -por ejemplo, en la figura 1 se muestran dos ocurrencias del T-pattern, de nivel 2 y de 4 clusters: ((AB)(CD)))-; o también entre T-patterns simples (AB) y un Evento-tipo (Et), generándose un T-pattern de nivel 2 y de 3 clusters: ((AB)Et).

Este proceso continúa hasta que THEME ha analizado todos los niveles posibles. De esta forma, los T-patterns van creciendo en complejidad (niveles y número de clusters). Entre los T-patterns de mayor nivel y/o número de clusters y los T-patterns simples (de primer nivel de relación y de 2 clusters), se detectan T-patterns de niveles y número de clusters intermedios, que constituyen porciones de estos T-patterns más complejos. Es lo que se conoce como degradación, que ha sido escasamente estudiada (Anguera, 2005) y que se constituye en el objetivo metodológico del presente trabajo. Para abordar esta cuestión, se han utilizado los registros correspondientes a un diseño observacional que pretende profundizar en el conocimiento de la composición de las secuencias ofensivas eficaces en el fútbol sala.

\section{La eficacia ofensiva del fútbol sala con un enfoque dinámico}

El análisis de la eficacia táctica con principios de auto-organización puede aumentar el rendimiento deportivo (Araújo, Davids y Hristovski, 2006; Araújo y Davids, 2009; Balagué y Torrents, 2011), pero su éxito depende de la comprensión y ejecución no lineal de los parámetros de coordinación y transición que aparecen en el juego competitivo y que están influidos por algunos aspectos contextuales (Nevill, Atkinson y Hughes, 2008; McGarry, Anderson, Wallace, Hughes y Franks, 2002). Desde este enfoque se considera el comportamiento colectivo táctico como un proceso emergente de interacción entre las limitaciones individuales, del contexto en el que se desarrollan, y las características de la tarea que emergen en diferentes subfases colectivas y que condicionan las conductas funcionales y los patrones de juego del equipo (Travassos, Davids, Araújo y Esteves, 2013; Davids, Button y Bennet, 2008).

La utilización de esta perspectiva dinámica-ecológica ha hecho posible describir comportamientos deportivos, constatando relaciones entre los datos registrados con los resultados del rendimiento (Reed y Hughes, 2006); y alcanzar una mayor comprensión de las interacciones de colaboración-oposición del equipo y de sus jugadores desde una perspectiva más funcional (Travassos, et al., 2013).

En el fútbol sala por las características del terreno de juego de espacio reducido y de su reglamento, se precisa de un gran dominio de la técnica y también de los principios básicos de la táctica ofensiva. Además, por la intensidad en el juego requiere 
de una gran demanda física -tiene un componente anaeróbico muy elevado (Álvarez, Manero, Manonelles y Puente, 2004)- y también psicológica pudiendo afectar en el rendimiento del jugador (Rodrigues, et al. 2011). Éste en cada ataque se ve obligado a decidir con gran rapidez la acción correcta a realizar, tanto sea cuando éste tiene la posesión del balón, ejecutando básicamente habilidades técnicas como el control del balón, conducción, regate o pase. $\mathrm{O}$ sin la posesión del mismo, poniendo en práctica el desmarque de apoyo, desmarque de ruptura u ocupar el espacio vacío dejado por el equipo contrario y los compañeros del propio equipo (Cabedo, Battala y López, 2012). De esta manera se pone de manifiesto la necesidad de una buena coordinación entre las acciones de los miembros de un mismo equipo para conseguir una ventaja respeto al equipo contrario (Riera, 2005). Las interacciones espacio temporales que se suceden constantemente entre los jugadores y la posición del balón constituyen parte fundamental del éxito en las jugadas de ataque (Vilar, Araújo, Davids y Button, 2012; Vilar, Araújo, Davids y Travassos, 2012). Este tipo de interacciones también han sido estudiadas en otros deportes de equipo, como el baloncesto, desde la perspectiva dinámica ecológica (Bourbosson, Poizat, Saury y Seve, 2010).

Es por este motivo, aunque el fútbol sala sea un deporte joven, que se ha empezado a valorar este aspecto tan determinante en el juego. Algunas investigaciones de referencia acerca del análisis ofensivo, ya han aportado datos significativos al respecto como las que han sido realizadas por los autores Barbero, Granda y Soto (2004), Irokawa, Lima, Soares, Souza y Greco (2010), Travassos, Araújo, McGarry y Vilar (2011), Leite (2012, 2013) y Lapresa, Álvarez, Arana, Garzón y Caballero (2013).

El presente artículo pretende valorar la eficacia de las jugadas de ataque desde el momento que el equipo posee el balón hasta la finalización en gol. Para este propósito se ha analizado el equipo del F.C. Barcelona, ganador de la liga Española del 2013, durante los partidos de semifinales y final del Play Off.

\section{Método}

El presente trabajo se sustenta en la metodología observacional cuyo carácter científico está ampliamente avalado (Anguera, Blanco-Villaseñor y Losada, 2001). Ésta nos permite el estudio sistemático de los comportamientos espontáneos que suceden en el ámbito deportivo, siendo en este caso en el fútbol sala. Utilizamos un diseńo observacional (Anguera, et al., 2001; Anguera, Blanco-Villaseñor, Hernández-Mendo y Losada, 2011) nomotético, de seguimiento y multidimensional (N/S/M): nomotético, porque se trata de un estudio del conjunto de jugadores de un mismo equipo que se enfrentan a otros equipos adversarios; de seguimiento, pues se valora un conjunto de secuencias que pertenecen a los diferentes partidos disputados por el F.C. Barcelona en el Play Off final de la liga Española de Futsal 2012-13; y multidimensional, ya que está conformado por las diferentes dimensiones que corresponden a los criterios del instrumento de observación.

\section{Participantes}

En el presente estudio de investigación se ha utilizado un muestreo intencional (Anguera, Arnau, Ato, Martínez, Pascual, y Vallejo, 1995) para analizar la construcción de las secuencias ofensivas eficaces (que acaban en gol) de los partidos disputados por el F.C. Barcelona durante el Play Off final de la liga española de FUTSAL correspondiente a la temporada 2012-13. Concretamente se han escogido los tres partidos realizados en la semifinal contra el equipo el Pozo Murcia y los cuatro partidos de la final con el Inter Movistar, siendo un total de siete encuentros.

\section{Instrumento de observación}

El instrumento de observación SOFS (tabla 1) diseñado ad hoc, mediante un panel de expertos compuesto por entrenadores de primera división, está compuesto por una combinación de formato de campo y sistema de categorías (Anguera, Magnusson y Jonsson, 2007; Sautu, Garay y Hernández-Mendo, 2009) que contiene 8 criterios que detectan la evolución de la fase ofensiva y que contempla: el tiempo del partido, el marcador, la superioridad o inferioridad numérica de nuestro equipo, el inicio de la pelota en el juego, el tipo de acción realizada por el jugador construyendo el ataque, la finalización del ataque, la finalización del chut, la zona de chut (figura 2) (Lapresa, et al., 2013) y su consecuencia.

Tabla 1. Instrumento de observación SOFS

\begin{tabular}{ccl}
\hline CRITERIO & Código/Categoría & DESCRIPCIÓN \\
\hline \multirow{2}{*}{ TIEMPO DE JUEGO } & $(\mathrm{P} 1)$ & 1a parte (1-10 minutos de juego $)$ \\
& $(\mathrm{P} 2)$ & 1a parte (11-20 minutos de juego $)$ \\
& $(\mathrm{P} 3)$ & 2a parte (1-10 minutos de juego $)$ \\
IGUALDAD NUMÉRICA & $(\mathrm{P} 4)$ & 2a parte (11-20 minutos de juego $)$ \\
& $(\mathrm{MN})$ Igualdad numérica & El equipo juega con el mismo número de jugadores \\
& $(\mathrm{SN})$ Superioridad numérica & El equipo juega con diferente número de jugadores \\
\hline
\end{tabular}




\begin{tabular}{|c|c|c|}
\hline CRITERIO & Código/Categoría & DESCRIPCIÓN \\
\hline \multirow{6}{*}{$\begin{array}{l}\text { INICIO DE LA PELOTA EN } \\
\text { JUEGO }\end{array}$} & (PORT)/ Portero & El portero inicia jugada pasando el balón a un compañero \\
\hline & (BAND)/ Banda & Un jugador inicia jugada desde la línea de banda \\
\hline & (RECU)/ Recuperación pelota & El mismo jugador que ha robado el balón inicia jugada \\
\hline & (FAL)/ Falta & Inicio de jugada de falta del equipo contrario \\
\hline & (CORN)/ Córner & Se inicia la jugada desde uno de los dos córners \\
\hline & (MEDC)/ Medio campo & Inicio de jugada o inicio del partido desde el medio campo \\
\hline \multirow{5}{*}{$\begin{array}{c}\text { ACCIÓN DEL JUGADOR EN } \\
\text { PROGRESIÓN }\end{array}$} & (PASPO)/ Pasada al portero & El jugador de campo le pasa la pelota a su portero \\
\hline & (PAS)/ Pasada & El jugador de campo le pasa la pelota a otro jugador \\
\hline & $(\mathrm{CONT}) /$ Control & El jugador de campo hace dos toques y realiza otra acción \\
\hline & (REG)/ Regate & El jugador de campo supera a su marcador \\
\hline & (COND)/ Conducción & El jugador de campo realiza más de dos toques y se desplaza \\
\hline \multirow{5}{*}{ FINALIZACIÓN DEL ATAQUE } & (PERDB) /Pérdida balón & El jugador de campo provoca pérdida de la posesión del balón \\
\hline & (ERRO)/ Error reglamentario & El jugador de campo realiza una infracción reglamentaria \\
\hline & (FALEC)/ Falta equipo contrario & El equipo contrario realiza una falta \\
\hline & (POBAL)/ Posesión balón & El equipo contrario sigue con la posesión de balón \\
\hline & (CHUT)/ Chute a portería & El jugador de campo realiza un chute a portería \\
\hline \multirow{8}{*}{$\begin{array}{l}\text { CONSECUCIÓN DEL } \\
\text { LANZAMIENTO }\end{array}$} & (GOLC)/ Gol de campo & El jugador realiza el gol desde cualquier parte del campo \\
\hline & (GOLP)/ Gol de penalti & El jugador transforma el penalti \\
\hline & (GOLDP)/Gol doble penalti & El jugador transforma el doble penalti \\
\hline & (GOLF)/ Gol de falta & El jugador realiza el gol desde el punto de la falta \\
\hline & $(\mathrm{POST}) /$ Poste & El jugador envía la pelota a la portería \\
\hline & (FUER)/ Fuera & El jugador envía el balón fuera del campo \\
\hline & (INTPO)/Intercepción portero & El portero del equipo contrario intercede o bloca el balón \\
\hline & (INTDE)/Intercepción defensa & Uno de los defensas intercede el balón \\
\hline \multirow{12}{*}{ ZONA DE LANZAMIENTO } & $(\mathrm{Z} 10) /$ Zona 10 & Sector seguridad / corredor izquierdo \\
\hline & $(\mathrm{Z} 20) /$ Zona 20 & Sector seguridad / corredor central \\
\hline & $(\mathrm{Z} 30) /$ Zona 30 & Sector seguridad / corredor derecho \\
\hline & $(\mathrm{Z} 40) /$ Zona 40 & Sector creación -campo propio- / corredor izquierdo \\
\hline & $(\mathrm{Z} 50) /$ Zona 50 & Sector creación -campo propio- / corredor central \\
\hline & $(\mathrm{Z} 60) /$ Zona 60 & Sector creación-campo propio- / corredor derecho \\
\hline & (Z41)/ Zona 41 & Sector creación -campo rival- / corredor izquierdo \\
\hline & $(\mathrm{Z} 51) /$ Zona 51 & Sector creación -campo rival- / corredor central \\
\hline & $(Z 61) /$ Zona 61 & Sector creación -campo rival- / corredor derecho \\
\hline & $(\mathrm{Z} 70) /$ Zona 70 & Sector definición / corredor izquierdo \\
\hline & $(\mathrm{Z} 80) /$ Zona 80 & Sector definición / corredor central \\
\hline & (Z90)/ Zona 90 & Sector definición / corredor derecho \\
\hline \multirow{5}{*}{ MARCADOR } & (V3)/ Ventaja más 2 goles & El equipo atacante gana con una ventaja de más de 2 goles \\
\hline & (V12)/ Ventaja 1 o 2 goles & El equipo atacante gana con una ventaja entre 1 o 2 goles \\
\hline & (EMPA)/ Empate & El marcador está en empate a goles \\
\hline & (D12)/ Desventaja 1 o 2 goles & El equipo atacante pierde por 1 o 2 goles \\
\hline & (D3)/ Desventaja más 2 goles & El equipo atacante pierde por más de 2 goles \\
\hline
\end{tabular}


Figura 2. Zonificación del campo de fútbol-sala (Lapresa, et al., 2013).

Spatial sense of the attack

$20 \mathrm{~m}$.

\begin{tabular}{|c|c|c|c|c|}
\hline $\begin{array}{l}\text { Left safety area } \\
\text { (Zone 10) }\end{array}$ & $\begin{array}{l}\text { Left Creation area } \\
\text { own area } \\
\text { (Zone 40) }\end{array}$ & $\begin{array}{l}\text { Left Creation area } \\
\text { opponent's area } \\
\text { (Zone 41) }\end{array}$ & $\begin{array}{l}\text { Left Definition area } \\
\text { (Zone 70) }\end{array}$ & $6.25 \mathrm{~m}$ \\
\hline $\begin{array}{c}\text { Centre safety area } \\
\text { (Zone 20) }\end{array}$ & $\begin{array}{l}\text { Centre Creation area } \\
\text { own area } \\
\text { (Zone 50) }\end{array}$ & $\begin{array}{c}\text { Centre Creation area } \\
\text { opponent's area } \\
\text { (Zone 51) }\end{array}$ & $\begin{array}{c}\text { Centre Definition area } \\
\text { (Zone 80) }\end{array}$ & $7.5 \mathrm{~m}$. \\
\hline $\begin{array}{l}\text { Right safety area } \\
\text { (Zone 30) }\end{array}$ & $\begin{array}{c}\text { Right Creation area } \\
\text { own area } \\
\text { (Zone 60) }\end{array}$ & $\begin{array}{c}\text { Right Creation area } \\
\text { opponent's area } \\
\text { (Zone 61) }\end{array}$ & $\begin{array}{l}\text { Right Definition area } \\
\text { (Zone90) }\end{array}$ & $6.25 \mathrm{~m}$. \\
\hline
\end{tabular}

$10 \mathrm{~m}$

$10 \mathrm{~m}$.

$10 \mathrm{~m}$.

$10 \mathrm{~m}$.

$40 \mathrm{~m}$.

\section{Instrumento de registro}

La informatización del registro (Hernández-Mendo, LópezLópez, Castellano, Morales-Sánchez y Pastrana, 2012) ha sido realizada con el software libre y versátil LINCE v.1.0. (Gabín, Camerino, Anguera y Castañer, 2012) que nos ha permitido introducir los diferentes criterios y categorías del instrumento de observación (SOFS) en la misma pantalla del ordenador y poder visualizar las imágenes de los partidos y el resultado de la codificación de los observadores (figura 3).
Este programa aporta procedimientos de observación informatizados en metodología observacional que agiliza el proceso de registro al visualizar las imágenes del partido en la misma pantalla del ordenador y permitir codificarlas simultáneamente, verificar el control de la calidad del dato de los diferentes observadores (Kappa), y exportar los resultados para el análisis a otros programas (THEME 6, SDIS-GSEQ, SAS y Excel) en archivos de diferentes formatos (HernándezMendo, Castellano, Camerino, Jonsson, Blanco-Villaseñor, Lopes y Anguera, 2014).

Figura 3. Instrumento de registro LINCE (Gabín, et al., 2012).

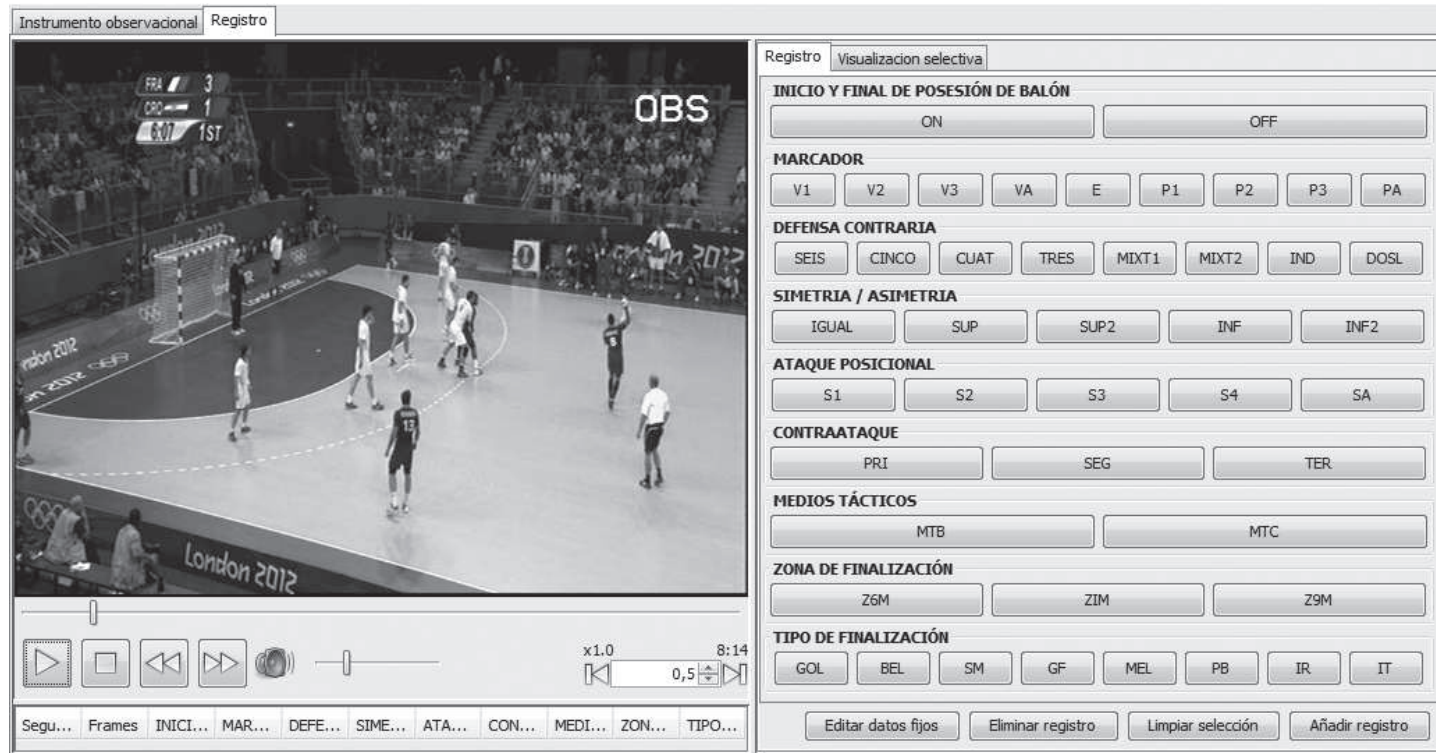




\section{Procedimiento observacional}

Una vez validado el instrumento (SOFS), se inició el entrenamiento de los dos observadores confeccionando un manual de observación en el que se definían los códigos y reglas del proceso observacional (Anguera y Hernández-Mendo, 2014). Se comprobó la calidad del dato con el cálculo del grado de concordancia o fiabilidad del registro de los dos observadores, utilizando el índice Kappa de Cohen (Cohen, 1960), en el que se obtuvo un valor de 0,96 en la fiabilidad interobservador comparando los dos registros. Las observaciones de los 7 enfrentamientos con el software LINCE (Gabín, et al., 2012) se exportaron, en formato .txt, al software THEME para el análisis de T-patterns.

\section{Análisis de datos}

De cara a la detección de T-patterns mediante el programa THEME, versión 6 , se han seleccionado los distintos parámetros de búsqueda. El primero de ellos ha sido el tipo de T-patterns free, que comporta la desactivación del fast requirement en todos los niveles (en los fast patterns el límite temporal inferior del intervalo crítico se fija en un valor igual a 0 , con lo cual los componentes del intervalo crítico tienden a ocurrir, relativamente, en rápida sucesión). En los free patterns el límite inferior del intervalo crítico se fija en la distancia más corta en que se presentan sus dos eventos constitutivos; por este motivo, no perdemos información proveniente de patrones cuyos componentes se relacionan a cierta distancia temporal, ya que siempre que estén dentro de la misma jugada los patrones detectados nos resultan relevantes. Además, se ha utilizado un nivel de significación de 0,005 , lo cual quiere decir que el porcentaje de aceptar un intervalo crítico debido al azar es de un $0,5 \%$. Se ha determinado una frecuencia de ocurrencia igual o mayor que 3; y para que los T-patterns sean seleccionados deben tener ocurrencia en, al menos, el 10\% de las secuencias (al menos en 3 de las jugadas constitutivas del muestreo observacional realizado). Por último consideramos relevante mencionar que se ha activado la herramienta de reducción de redundancias que aporta THEME, sin obtenerse ningún efecto, incluso bajo el parámetro que implica una mayor reducción.

Para clasificar los T-patterns en términos de simetría (véase figura 4) se ha utilizado el planteamiento expuesto en Anguera (2005).

Figura 4. Tipos de simetria en T-patterns (Anguera, 2005, p. 64).

\section{Central}

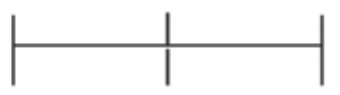

Axial

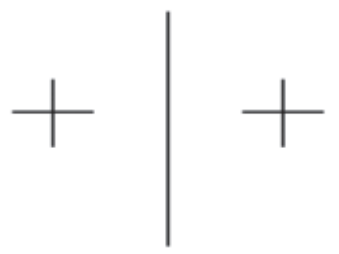

Mirror

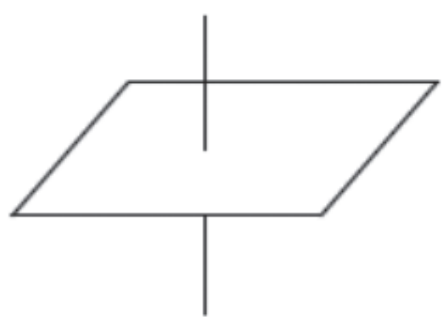

\section{Resultados}

En el muestreo observacional efectuado se han registrado 244 eventos. En total el número de agrupaciones de códigos diferentes (Eventos-tipo, en terminología THEME) ha sido de 86; lo que supone una frecuencia media de aparición de 2,83.

A continuación, en la tabla 2, se muestran los T-patterns detectados con los parámetros de búsqueda prefijados y su número de ocurrencias o frecuencia de aparición. Al respecto mencionar que, para facilitar el análisis de la degradación de

los T-patterns, se ha modificado el identificador del T-pattern de forma que el primer dígito se corresponde con el nivel del T-pattern y el segundo con el número de orden dentro del correspondiente nivel. De esta forma, los T-patterns de mayor nivel se han colocado a la izquierda de la tabla 2, pudiéndose seguir -en la misma fila (hacia la derecha)- la degradación de cada T-pattern de nivel superior en niveles inferiores.

Por su relevancia en el análisis de la degradación de los $T$ patterns, en la tabla 3 se procede a clasificar los T-patterns en términos de simetría. 
Tabla 2. T-patterns detectados con los parámetros de búsqueda prefijados. Siendo ID= identificador del T-pattern, N= número de ocurrencias del T-pattern. A la izquierda los T-patterns de mayor nivel y su posterior degradación hacia niveles inferiores (hacia la derecha).

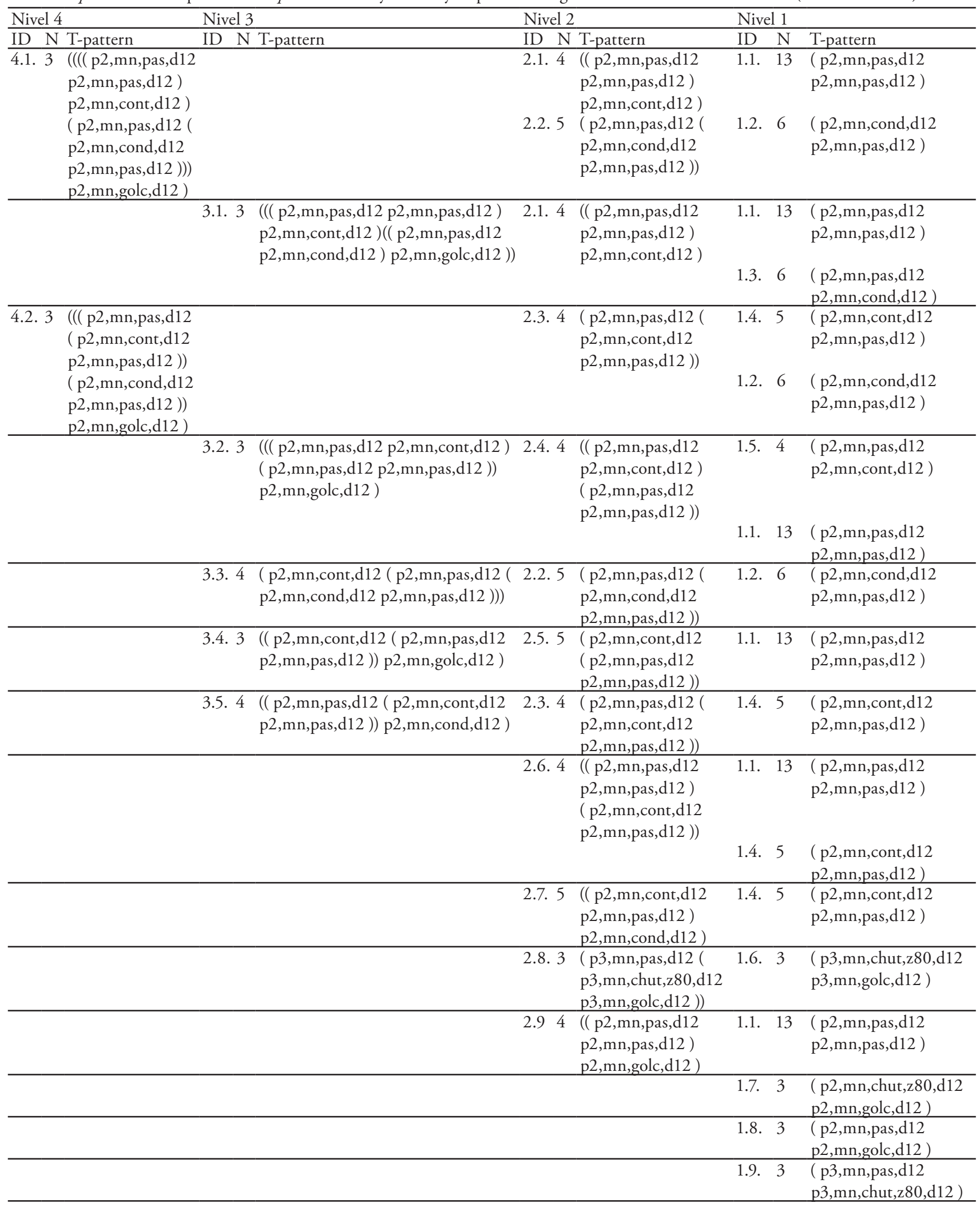


Tabla 3. T-patterns detectados, con su correspondiente degradación, en términos de simetría/asimetría. Siendo ID= identificador; Nc= número de clusters; A= Estructura Asimétrica; Simetría central $=\longmapsto|-|$; Simetría axial $=+\mid+$; Simetría especular $=+$. En negrita subestructuras que no constituyen T-pattern (de nivel 2) en el proceso de degradación.

\begin{tabular}{|c|c|c|c|c|c|c|c|}
\hline \multicolumn{2}{|l|}{ Nivel 4} & \multicolumn{2}{|l|}{ Nivel 3} & \multicolumn{2}{|l|}{ Nivel 2} & \multicolumn{2}{|l|}{ Nivel 1} \\
\hline ID $\mathrm{Nc}$ & Tipo & ID $\mathrm{Nc}$ & Tipo & ID $\mathrm{Nc}$ & Tipo & ID $\mathrm{Nc}$ & Tipo \\
\hline \multirow[t]{4}{*}{4.17} & $\mathrm{~A}\{$ Especular $\}=\mathrm{A}\{[$ Central $]$ & & & 2.1 .3 & [Central] A & 1.1. 2 & Central \\
\hline & A - A $[$ Central $]\}$ & & & 2.2 .3 & A [Central] & 1.2. 2 & Central \\
\hline & & 3.1 .6 & Axial $=[$ Central $]$ A $-[$ Cen- & 2.1 .3 & [Central] A & 1.1. 2 & Central \\
\hline & & & tral] A & & & 1.3. 2 & Central \\
\hline \multirow{15}{*}{4.26} & Especular $=$ A [Central $]-$ & & & 2.3 .3 & A [Central] & 1.4 .2 & Central \\
\hline & {$[$ Central $] \mathrm{A}$} & & & & & 1.2. 2 & Central \\
\hline & & 3.2 .5 & $\{$ Axial $\} A=\{[$ Central $]-$ & 2.4 .4 & Axial $=[$ Central $]-[$ Central $]$ & 1.5 .2 & Central \\
\hline & & & $[$ Central $]\}$ A & & & 1.1. 2 & Central \\
\hline & & 3.3 .4 & A A [Central] & 2.23 & A [Central] & 1.2 .2 & Central \\
\hline & & 3.4 .4 & Especular= A [Central] A & 2.5 .3 & A [Central] & 1.1. 2 & Central \\
\hline & & 3.5 .4 & Especular $=\mathrm{A}$ [Central] A & 2.3 .3 & A [Central] & 1.4. 2 & Central \\
\hline & & & & 2.6 .4 & Axial $=[$ Central $]-[$ Central $]$ & 1.1. 2 & Central \\
\hline & & & & & & 1.4. 2 & Central \\
\hline & & & & 2.7 .3 & [Central] A & 1.4. 2 & Central \\
\hline & & & & 2.8 .3 & A [Central] & 1.6. 2 & Central \\
\hline & & & & 2.9. 3 & [Central] A & 1.1. 2 & Central \\
\hline & & & & & & 1.7. 2 & Central \\
\hline & & & & & & 1.8. 2 & Central \\
\hline & & & & & & 1.9. 2 & Central \\
\hline
\end{tabular}

\section{Discusión}

\section{En relación a la degradación de los T-patterns}

A continuación se procede a la discusión de los resultados obtenidos, con miras a satisfacer el objetivo metodólogico de analizar el proceso de degradación de los T-patterns. Al respecto, la discusión del proceso de degradación de los T-patterns se sustenta en tres líneas argumentales: a) la continuidad en el orden (clusters contiguos) de las agrupaciones de códigos que configuran el T-pattern de superior nivel; b) el descenso progresivo del número de clusters constitutivos de los T-patterns; c) la evolución de la simetría/asimetría de los T-patterns. Estas líneas argumentales, a pesar de que se aborden por separado, se encuentran interrelacionadas entre sí y evidentemente condicionadas por el proceso de detección por niveles de T-patterns (de nivel 1 -simples- a nivel n) descrito en el apartado de introducción.

\section{a) Continuidad-discontinuidad de los clusters} constitutivos de los T-patterns de mayor nivel

Si analizamos la degradación de los T-pattens detectados (tabla 2), podemos comprobar cómo, en todos los casos, la progresiva degradación se produce manteniendo clusters consecutivos del T-pattern de nivel superior. Dicho de otra forma, no se han detectado T-patterns degradados que incorporen clusters de códigos que, en los T-patterns de nivel superior, se encuentren intercalados. En todos los casos se pierden clusters de la periferia del T-pattern, ya sea: un Evento-tipo; o T-patterns (en negrita, en la tabla 3) constituidos por un T-pattern simple y un Evento-tipo: ((AB)Et).

\section{b) Descenso progresivo del número de clusters de los T-patterns}

En relación a la degradación en función del número de clusters que constituyen el T-pattern, decir que, en el presente trabajo, cuando un T-pattern tiene un número de clusters impar (7, 5 y 3 -en nuestro caso-), pierde el cluster correspondiente al Evento-tipo. De esta forma el T-pattern de 7 clusters (véase figura 5) se convierte en dos paquetes de 3 clusters y nivel 2 $(\mathrm{Et}(\mathrm{AB}))$ y $((\mathrm{AB}) \mathrm{Et})$. Por su parte, el T-pattern de 5 clusters, pierde un Evento-tipo y se convierte en un T-pattern de 4 clusters y nivel $2((\mathrm{AB})(\mathrm{CD}))$; que posteriormente se degradará en dos patrones simples (AB) y (CD). Mientras que, todos los T-patterns de 3 clusters, se degradan de ((AB)Et) a (AB).

En relación a los T-patterns con número de clusters par, el detectado de 6 clusters (véase figura 6) se degrada en dos paquetes de 3 clusters (Et (AB)) y ((AB)Et). Por su parte, los T-patterns de 4 clusters presentan diferentes modalidades de degradación: los de nivel 3 se degradan, en todos los casos, a (Et (AB)); mientras que, los de nivel $2((\mathrm{AB})(\mathrm{CD}))$, se degradan a $(\mathrm{AB})$ y $(\mathrm{CD})$. 
c) Degradación de los T-patterns en términos de simetríalasimetría

A continuación se procede a abordar la cuestión de la degradación en términos de simetría/asimetría, a partir de Anguera (2005). La degradación de los T-patterns asimétricos se caracteriza (véase tabla 3) por la pérdida de su cluster asimétrico exterior -el correspondiente al Evento-tipo-, para finalizar (en puridad "iniciar" -conforme al mencionado proceso de detección de T-patterns seguido por THEME-) en un T-pattern simple. Ésta es la razón por la que el T-pattern con ID = 4.1. (véase tabla 2 y figura 5), se degrada en dos T-patterns de nivel 2 (ID = 2.1. y 2.2.). En los T-patterns con ID = 4.2 y 3.1. (véase figura 6), la degradación se produce perdiendo, además del cluster asimétrico (Evento-tipo), un T-pattern simple -es decir, perdiendo la estructura ((AB)Et)-.

En el caso de las estructuras con simetría axial o especular -constituidas en el presente trabajo por dos subestructuras-, al degradarse dan lugar a los correspondientes dos T-patterns de nivel inferior o a uno sólo de ellos (como ya hemos mencionado que ocurre en los T-patterns con ID= 4.2 y 3.1.).

En todos los casos, el T-pattern de nivel superior acaba su degradación en los T-patterns simples (simetría central) correspondientes. Este hecho es inherente al funcionamientoproceso de detección por niveles (de nivel inferior a superior) que utiliza THEME. Dicho de otra forma los T-patterns de simetría central configuran, en sus posteriores relaciones entre sí o con otros eventos, las relaciones de nivel superior.

Figura 5. Dendogramas que reflejan la degradación del T-pattern con ID=4.1., de 7 clusters, 4 niveles y con estructura: A $\{$ Especular $\}=$ A $\{[$ Central $]$ A - A [Central $]\}$.

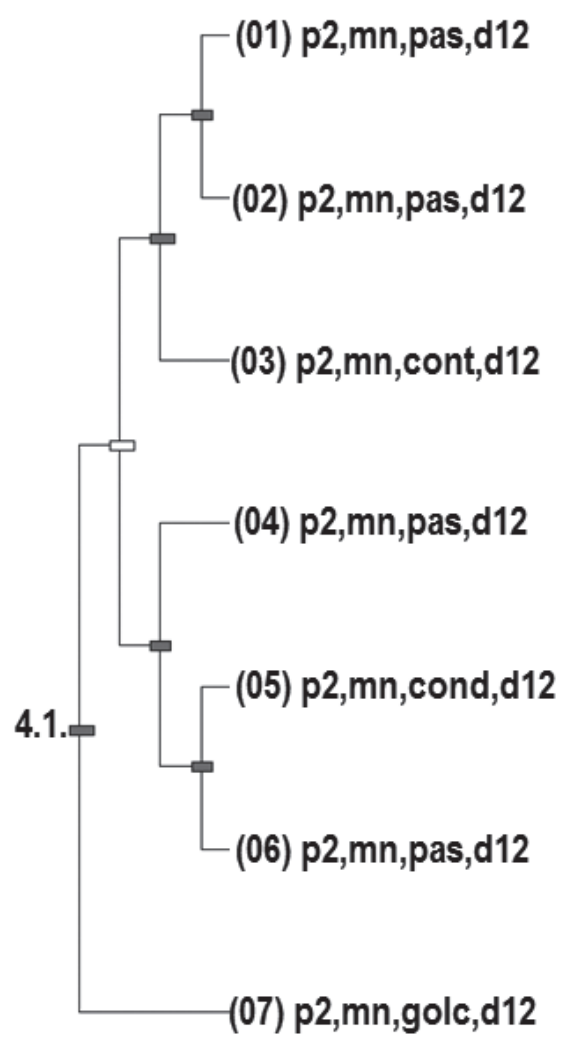

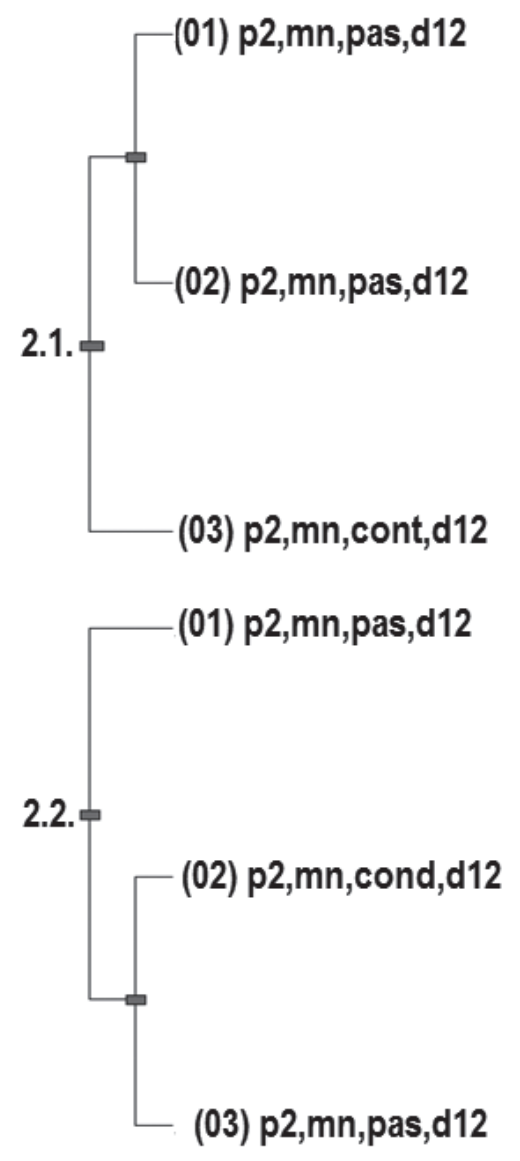
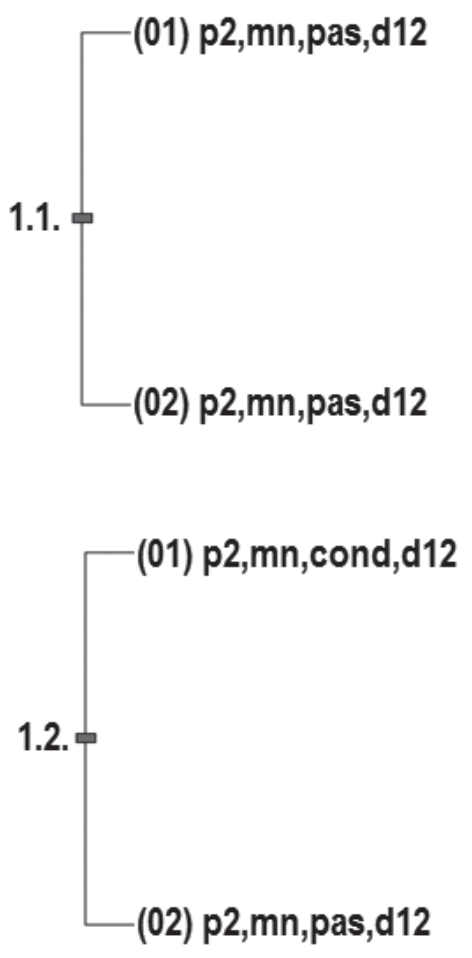
Figura 6. Dendogramas que reflejan la degradación del T-pattern con ID=3.1., de 6 clusters, 3 niveles y simetría Axial= [Central $]$ A [Central] A.

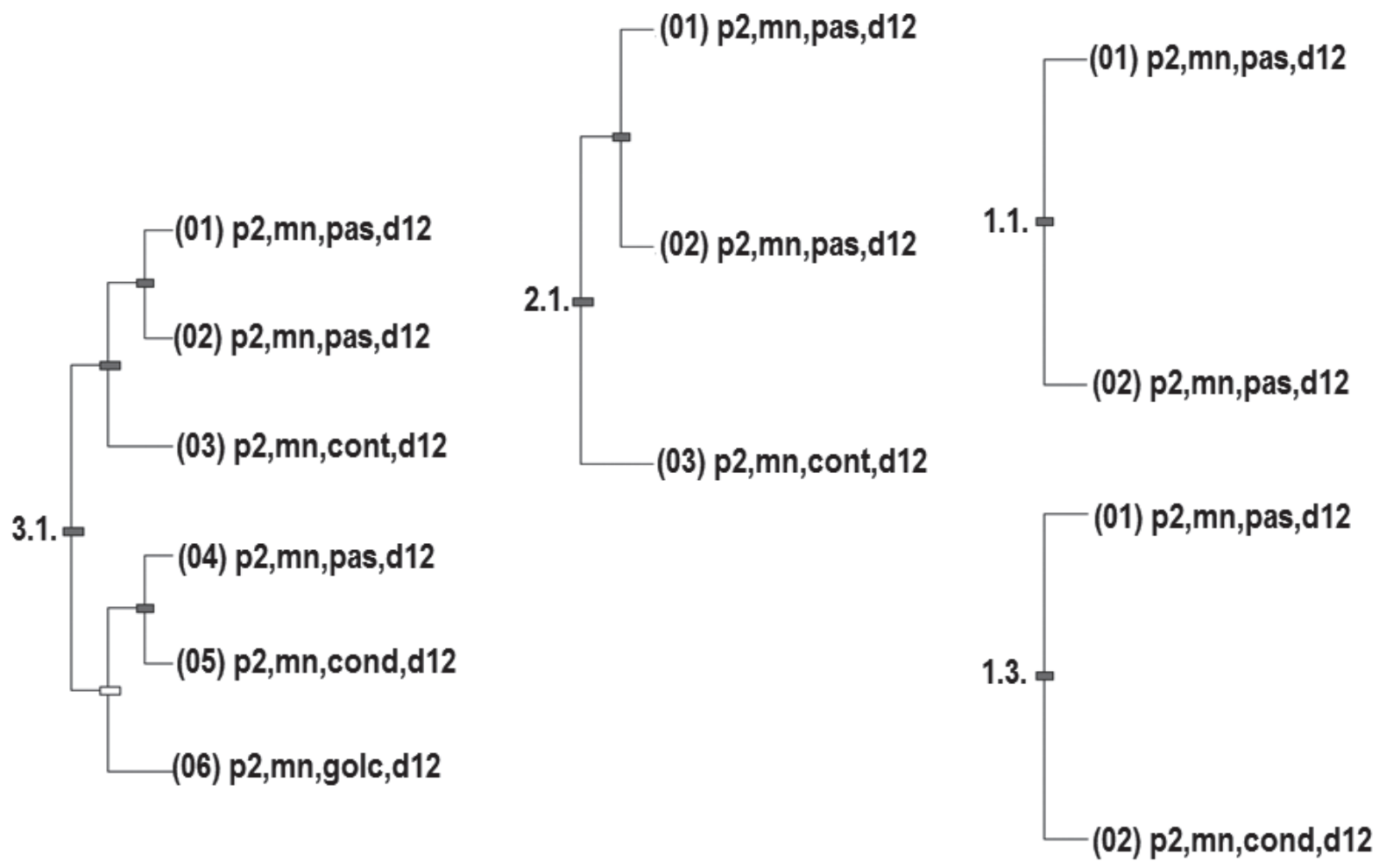

En relación a las secuencias ofensivas eficaces

En relación a las jugadas-secuencias en las que acontecen las ocurrencias de los T-patterns detectados (tabla 2), pueden agruparse en dos grandes conjuntos: a) todos aquellos T-patterns que se refieren al $2^{\circ}$ cuarto de juego (p2), tienen lugar en las secuencias con número de orden 4, 5, 7 y 9; b) en relación al tercer cuarto de juego (p3) se han detectado los T-patterns con $\mathrm{ID}=2.8,1.6$. y 1.9 .

La mejor forma de delimitar la estructura de las secuencias ofensivas que finalizan en gol en el segundo cuarto, resulta de interpretar el T-pattern con ID=4.1. -que tiene lugar en las jugadas que, consideradas sucesivamente, tienen los números de orden cuarto, quinto y séptimo- y el T-pattern con $\mathrm{ID}=1.7$, presente en las jugadas cuarta, quinta, séptima y novena, y que complementa al T-pattern de mayor número de clusters al incluir el evento chut. El T-pattern 4.1. (figura 5), ocurre durante los últimos diez minutos de la primera parte (P2), en igualdad numérica por los dos equipos (MN) y con una desventaja en el marcador entre uno y dos goles (D12). En estas circunstancias se refleja la secuencia constituida por las diferentes acciones coordinadas entre los jugadores en el siguiente orden: se inicia con un pase (PAS), seguido de otro pase (PAS), un control del balón (CONT), otro pase a un compañero (PAS), seguido de una conducción del mismo jugador (COND), realizando este un pase (PAS), antes de lanzar (CHUT) a portería -desde zona 80, tal y cómo nos indica el T-pattern con ID=1.6.- para finalizar en gol (GOLC).

El T-pattern con ID = 2.8 (que incluye las agrupaciones de códigos del T-pattern degradado 1.6. y del 1.9.), refleja una secuencia más corta (3 clusters) en el tercer cuarto, en el que tras un pase (PAS) en terreno ofensivo, se realiza el lanzamiento (CHUT) que finaliza en gol (GOLC), desde la zona 80.

En ambos casos, los resultados están en sintonía con los obtenidos por Álvarez, Manero, Manonelles y Puente (2004), Martín (2009) y Lapresa, et al. (2013), en lo relativo a la zona que predispone la eficacia del lanzamiento y la forma en la que se alcanza dicha zona mediante juego combinativo. Este juego combinativo se caracteriza por la dificultad que supone realizar más de dos pases seguidos sin aliviar el juego -mediante un control o conducción- antes de lanzar a portería.

Aplicaciones prácticas

Las aplicaciones se corresponden con los dos objetivos planteados en el presente trabajo. Por un lado, en la vertiente metodológica, orienta al investigador y/o profesional que se encuentra con una multiplicidad de T-patterns que resultan 
difíciles de interpretar -por las múltiples posibilidades de navegación que caben entre ellos-, en la comprensión de los resultados obtenidos mediante THEME, facilitando el entendimiento del proceso de degradación de los T-patterns detectados (de complejos a simples). Además, en el ámbito del futbol sala, se evidencian las posibilidades que los T-patterns ofrecen en el conocimiento de la construcción de las secuencias ofensivas eficaces.

Notas: ${ }^{1}$ Al gráfico que muestra la estructura de conjunto (T-pattern tree graph ó binary clustering tree) lo denominamos "dendograma", mientras que a las agrupaciones de códigos que, como resultado del análisis, se manifiestan en cada una de las ramas del gráfico en forma de árbol, la denominamos "cluster", y consiste en una agrupación de códigos obtenida estadísticamente en el proceso de análisis.

Becas, ayudas o soporte financiero: Este artículo se ha realizado con el apoyo y financiación de: Grup de recerca $i$ innovació en dissenys (GRID). Tecnologia $i$ aplicació multimedia $i$ digital als dissenys observacionals. Grupo Consolidado de la Generalitat de Catalunya [Referencia: 2014 SGR 971]. Asimismo, ha recibido el apoyo del Proyecto I+D+I: Observación de la interacción en deporte y actividad física: Avances técnicos $y$ metodológicos en registros automatizados cualitativos-cuantitativos. Secretaría de Estado de Investigación, Desarrollo e Innovación del Ministerio de Economía y Competitividad [Referencia: DEP2012-32124].

\section{Referencias}

1. Álvarez, J., Manero, J., Manonelles, P. y Puente, J. (2004). Analysis of the offensive actions resulting in goal of professional league of Spanish Futsal. Revista de entrenamiento deportivo, 18 (4), 27-32.

2. Anguera, M.T. (2005). Microanalysis of T-patterns. Analysis of simmetry/assimetry in social interaction. In L. Anolli, S. Duncan, M. Magnusson y G. Riva (Eds.), The hidden structure of social interaction. From Genomics to Culture Patterns (pp. 51-70). Amsterdam: IOS Press.

3. Anguera, M.T. y Hernández-Mendo, A. (2014). Metodología observacional y psicología del deporte: Estado de la cuestión. Revista de Psicología del Deporte, 23(1), 103-109.

4. Anguera, M.T., Blanco-Villaseñor, A. y Losada, J.L. (2001). Diseños observacionales, cuestión clave en el proceso de la metodología observacional. Metodología de las Ciencias del Comportamiento, 3(2), 135-160.

5. Anguera, M.T., Blanco-Villaseñor, A., Hernández-Mendo, A. y Losada, J. L. (2011). Diseños observacionales: ajuste y aplicación en psicología del deporte. Cuadernos de Psicología del Deporte, 11(2), 63-76.

6. Anguera, M.T., Arnau, J., Ato, M., Martínez, R., Pascual, J. y Vallejo, G. (1995). Métodos de investigación en psicología. Madrid: Síntesis.

7. Anguera, M.T., Magnusson, M.S. y Jonsson, G.K. (2007). Instrumentos no estándar. Avances en medición, 5(1), 63-82.

8. Araujo, D., Davids, K. y Hristovski, R. (2006). The ecological dynamics of decision making in sport. Psychology of Sport and Exercise, 7(6), 653-676.

9. Araújo, D. y Davids, K. (2009). Ecological approaches to cognition and action in sport and exercise: Ask not only what you do, but where you do it. International Journal of Sport Psychology, 40, 5-37.

10. Balagué, N. y Torrents, C. (2011). Complejidad y deporte. Barcelona: Inde.

11. Barbero, J.C., Granda, J. y Soto, V.M. (2004). Análisis de la frecuencia cardíaca durante la competición en jugadores profesionales de fútbol sala. Apunts: Educación Física y Deportes, 77, 71-78.

12. Bourbousson, J., Poizat, G., Saury, J. y Seve, C. (2010). Team coordination in basketball: Description of the cognitive connections among teammates. Journal of Applied Sport Psychology, 22(2), 150-166.

13. Cabedo, J., Batalla, A. y López, V. (2012). ¿Cómo se enseña y cómo se aprende a ser competente en un deporte? Estrategias de enseñanza. Barcelona: Inde.

14. Camerino, O., Chaverri, J., Anguera, M.T. y Jonsson, G.K. (2012). Dynamics of the game in soccer: Detection of T-patterns. European Journal of Sport Science, 12(3), 216-224.

15. Camerino, O., Prieto, I., Lapresa, D., Gutiérrez-Santiago, A. e Hileno, R. (2014). Detección de T-patterns en la observación de deportes de combate. Revista de Psicología del Deporte, 23(1), 147-155.
16. Cohen, J. (1960). A coefficient of agreement for nominal scales. Educational and Psychological Measurement, 20, 37-46.

17. Davids, K., Button, C. y Bennet, S. (2008). Dynamics of skill acquisition. Champaign IL: Human Kinetics.

18. Gabín, B., Camerino, O., Anguera, M. T. y Castañer, M. (2012). Lince: multiplatform sport analysis software. Procedia-Social and Behavioral Sciences, 46, 4692-4694.

19. Hernández-Mendo, A., Castellano, J., Camerino, O., Jonsson, G.K., Blanco-Villaseñor, A., Lopes, A. y Anguera, M.T. (2014). Programas informáticos de registro, control de calidad del dato, y análisis de datos. Revista de Psicología del Deporte, 23(1), 111-121.

20. Hernández-Mendo, A., López-López, J.A., Castellano, J., MoralesSánchez, V. y Pastrana, J.L. (2012). Hoisan 1.2: Programa informático para uso en metodología observacional. Cuadernos de Psicología del Deporte, 12(1), 55-78.

21. Irokawa, G.N., Lima, M.R., Soares, V.O., Aburachid, L.M., Souza, P.R. y Greco, P.J. (2010). Caracterização das circunstâncias e setores de finalização do jogo de futsal:um estudo da fase final da copa do mundo de futsal-FIFA 2008. Revista EFDeportes.com, 15 (144).

22. Lapresa, D., Álvarez, L., Arana, J., Garzón, B. y Caballero, V. (2013): Observational analysis of the offensive sequences that ended in a shot by the winning team of the 2010 UEFA Futsal Championship. Journal of Sport Sciences, 31(15), 1731-1739.

23. Lapresa, D., Arana, J., Anguera, M.T. y Garzón, B. (2013). Comparative analysis of the sequentiality using SDIS-GSEQ and THEME: a concrete example in soccer. Journal of Sport Sciences, 31(15), 16871695.

24. Leite, W.S. (2012). Determination of offensive coefficients in high performance futsal. Pamukkale Journal of Sport Sciences, 3(3), 78-89.

25. Leite, W.S. (2013). Determination of offensive coefficients in high performance futsal. Serbian Journal of Sport Sciences, 7(4), 167-172.

26. McGarry, T., Anderson, D., Wallace, S., Hughes, M. y Franks, I. (2002). Sport competition as a dynamical self-organizing system. Journal of Sport Sciences, 20(10), 771-781.

27. Magnusson, M.S. (1996). Hidden real-time patterns in intra- and inter-individual behavior. European Journal of Psychological Assessment, 12(2), 112-123.

28. Magnusson, M.S. (2000). Discovering hidden time patterns in behavior: T-patterns and their detection. Behavior Research Methods, Instruments \& Computers, 32(1), 93-110.

29. Magnusson, M.S. (2005). Understanding social interaction: Discovering hidden structure with model and algorithms. In L. Anolli, S. 
Duncan, M. Magnusson \& G. Riva (Eds.), The hidden structure of social interaction. From Genomics to Culture Patterns (pp. 4-24). Amsterdam: IOS Press.

30. Magnusson, M.S. (2006). Structure and Communication in Interaction. In G. Riva, M.T. Anguera, B.K. Wiederhold \& F. Mantovani (Eds.), From Communication to Presence: Cognition, Emotions and Culture Towards the Ultimate Communicative Experience (pp. 127-146). Amsterdam: IOS Press.

31. Martín, J. (2009). ¿Dónde radica el error del gol en el fútbol sala? Análisis. Revista Internacional de deportes colectivos, 2, 36-57.

32. Nevill, A., Atkinson, G. y Hughes, M. (2008). Twenty-five years of sport performance research in the journal of sports sciences. Journal of Sports Sciences, 26(4), 413.

33. Reed, D. y Hughes, M. (2006). An exploration of team sport as a dynamical system. International Journal of Performance Analysis in Sport, 6(2), 114-125.

34. Riera, J. (2005) Habilidades en el deporte. Barcelona: Inde.

35. Rodrigues, V.M., Ramos, G.P., Mendes, T.T., Cabido, C.E.T., Melo, E.S., Condessa, L.A., ... Silami-Garcia, E.I. (2011). Intensity of oficial futsal matches. Journal of strength and conditioning research, 25(9), 2482-2487.
36. Sautu, L.M., Garay, J.O. y Hernández-Mendo, A. (2009). Observación y análisis de las interacciones indirectas en el baloncesto ACB. Cuadernos de Psicología del Deporte, 9 (Suplemento), 69.

37. Travassos, B., Araújo, D., McGarry, T. y Vilar, L. (2011). Interpersonal coordination and ball dynamics in futsal (indoor football). Human Movement Science, 30, 1245-1259.

38. Travassos, B., Davids, K., Araújo, D. y Esteves, P. T. (2013). Performance analysis in team sports: Advances from an Ecological Dynamics approach. International Journal of Performance Analysis in Sport, 13(1), 83-95.

39. Vilar, L., Araújo, D., Davids, K. y Button, C. (2012). The role of ecological dynamics in analysing performance in team sports. Sports $\mathrm{Me}$ dicine, 42(1), 1-10.

40. Vilar, L., Araújo, D., Davids, K. y Travassos, B. (2012). Constraints on competitive performance of attacker-defender dyads in team sports. Journal of Sports Sciences, 30(5), 459-469.

41. Zurloni, V., Cavalera, C., Diana, B., Elia, M. y Jonsson, G. (2014). Detecting regularities in soccer dynamics: A T-pattern approach. Revista de Psicología del Deporte, 23(1), 157-164. 